\section{Debriefing and post traumatic stress disorder}

Sir - There is considerable confusion as to the merits of psychological debriefing after a traumatic event. From the initial optimism that it would be preventative for the development of post traumatic stress disorder, to the findings of the Cochrane Report. ${ }^{1}$ The Cochrane Report concluded that there was a failure to demonstrate any benefit from psychological debriefing for the prevention of post traumatic stress disorder after traumatic incidents. It also stated that compulsory debriefing of victims of trauma should cease. The mandatory debriefing of the 55,000 US police and firefighters involved in the aftermath of the September 11 incident has been criticised ${ }^{2}$ as a waste of time, and that it will not prevent anyone from developing post traumatic stress disorder.

There are implicit difficulties in conducting rigorous randomised controlled trials of group debriefing, and such trials may be unachievable. ${ }^{2}$ The problems with this kind of research are: that psychological debriefing has not been clearly defined; the training of debriefers has not been made clear; the length of intervention has not been consistent; and there is no standardisation of the timing of the intervention. That debriefing is usually meant to be a group process is missed by those who debrief individually, and who use their results as a comparator for all forms of debriefing.

There has probably been an over-emphasis on the prevention or the amelioration of post traumatic stress disorder in these studies. This has been to the detriment of assessing for co-morbid psychopathologies such as alcohol abuse, ${ }^{3}$ anxiety, phobic reactions, depression and adjustment reactions.

It is well recognised that major trauma is associated with a potential for developing a variety of psychiatric illnesses. The predisposing personality factors have not yet been properly studied, though the concept of 'hardiness' ${ }^{4}$ has been proposed as a preventative factor. There is an argument that there is a 'dose-response' relationship between the dose of the trauma and the psychiatric response. This is being questioned, in that it is also being proposed that it is the perception of the dose of the trauma that matters, rather than the real amount of trauma.

Then we have difficulties in determining the contributing factors of previous trauma when compared to those of the index event. This is particularly pertinent, as those most likely to receive debriefing are rescue workers, police, and combatants. These are the groups who are most likely to have been exposed to previous traumatic events. Has their history of previous trauma left them 'brittle' to the development of post traumatic psychological sequelae?

Common sense has to win out! We will probably never be able to create a study that will tell us whether we are doing good or harm with psychological debriefing. There are just too many variables to confound such studies. In these circumstances we must fall back on dose-response, and continue our search for vulnerability factors in the recipient to developing psychiatric illness following major trauma.

Events involving multiple casualties are more likely to be an important cause of psychological trauma among the survivors and the rescue personnel. ${ }^{5}$ In such situations psychological debriefing is of likely benefit. It is important that it is done as a group process. This allows a recognition of the universality of emotional response, and if done correctly an acceptance of emotions in an understanding environment with those who have endured similar trauma.

There are essential rules of engagement which must be adhered to. These include: never getting in the way of the rescue operations; only to intervene when the victims are ready to participate mentally as well as physically; never interfere with, or delay the official investigation into the events. This is very important if there is to be a police investigation, as the debriefing process can lead to an inappropriate rehearsal by the group of the testimony that the police eventually receive.

In this regard timing of intervention is important. There was a belief in the 1990 s that early intervention was most beneficial. ${ }^{6}$ Mitchell initially recommended that it should occur 24 to 72 hours after the incident, ideally within three to eight hours, using a defusing process of about one hour. Anyone who has been involved in psychological debriefing after a major disaster knows that this is unrealistic.

Rose and Bisson ${ }^{7}$ highlighted the difficulties of drawing conclusions about the effectiveness of early psychological debriefing, given the paucity of methodologically sound studies, differences in the type of incidences, type of victims and type of interventions. Currently there is no empirical evidence to indicate the optimal time to debrief. ${ }^{8}$

The next question is whether we are, in fact, doing harm by intervening with a formal debriefing method. Just because we mean well does not mean that we are not doing harm. The most likely danger is to lead to scapegoating of an individual or group of individuals. A training in group psychotherapy is of benefit in this kind of situation. In certain situations individual debriefing may be the preferred method.

We can also be placing people into a victim role, rather than into the role of a survivor, with all the connotations that these roles have. From the outset the survivor role needs to be emphasised, and the educational element needs to demonstrate the normal emotional response that a survivor may have.

Our next difficulty is to decide whether to intervene in situations of minor or moderate trauma. Indeed, we are faced with a problem in defining what is minor and what is moderate trauma. We are back to that thorny question of perception. There is a mixture of personal, social, experiential and cultural elements in defining the severity of the trauma, and the emotional response to it. What may be a major trauma to a person in Dublin, may be a minor or moderate trauma to a person in Mogadhisu. This is one area that cannot be delineated with any certainty.

There would appear to be a decreasing benefit from psychological debriefing as we go from major trauma to minor trauma. If you were to place road traffic accidents under the heading of moderate trauma, negative findings for psychological debriefing are more commonplace. ${ }^{9}$ Similarly in studies of burns victims. ${ }^{10}$ The problem is that the negative finding in these studies have also led to a negative view of intervention in major trauma, such as when multiple casualties occur.

I am of the opinion that any purpose is served by medicalising the debriefing process. It is of benefit to have a psychiatrist available in the background for those rare 
occasions when an event has so disturbed an individual's psyshe that short term pharmacological intervention is required. Also, the knowledge that the debriefers have a senior mental health professional available to them is a source of confidence and reassurance to the them.

I believe that the best debriefers are those who come from within the 'at risk' organisation, who are both well respected, and experienced, and with whom those who receive debriefing can identity. In many ways these are the people who are role models within the organisation. Their acknowledgement of the normal human emotions after a traumatic event carries weight with the potentially traumatised.

The utilisation of external debriefers, no matter how well qualified, will suffer from a difficulty in acceptance from those undergoing the debriefing process. There is the added difficulty in that those being debriefed will view the process as a 'box ticking exercise', and not related to their specific needs. If they know the debriefers and hold them in high regard, it is more likely that they will take the process seriously and benefit from it.

Overall it has been my observation that those who have been debriefed, like it. They express the opinion that after a traumatic event, it is a recognition that the organisation cares about their emotional well-being. How many times have we all heard anger being projected at the authorities from a perception that they did not 'care'. It is possible that this is a contributing factor to the anger that is evident in many suffering from post traumatic stress disorder. It is for this reason more than any other, that I believe we should not ditch post traumatic debriefing, and that we use it wisely and with debriefers with whom people can identity.

Whether or not debriefing prevents post traumatic stress disorder, we will never know for sure. There are just too many variables to measure. So we can continue to expect conflicting results, and the debate as to its harms and benefits will run on and on.

\section{John Tobin \\ Consultant psychiatrist St Bricin's Military Hospital, Dublin, Ireland.}

\footnotetext{
References

I. Wessely S, Rose S, Bisson J. Brief psychological interventions (debriefing) for trauma related symptoms and the prevention of post traumatic stress disorder: Cochrane Database, Syst Rev 2000

2. Lavie P. New England Journal of Medicine 2001: 343: 1825-32.

3. Deahl M. Psychological debriefing: controversy and challenge: Aus NZ Journal of Psychiatry 2000; 34(6): 929-39.

4. Deahl M, Srinivasan M, Jones $N$ et al. Preventing psychological trauma in soldiers: the role of operational stress training and psychologkal debriefing. British Journal of Medical Psychology 2000; Mar '73, 77-85.

5. Waysman M Schwaimald J, Solomon Z. Hardiness: an examination of its relatioship with positive and negative long term changes following trauma. Journal of Traumatic Stress 2001; 14:(3), 531-48.

6 . Wood D, Cowan M, Crisis intervention following disasters: Are we doing enough? Amer J Emerg Med: 1991 Nov; 9, (6): 598-602.

7. Mitchell J. Everly G. Critical incident stress debriefing: an operational manual for the prevention of traumatic stress among emergency service and disaster workers; (2nd edition); Chevron Publishing Corporation.

disaster workers; (2nd edition); Chevron Publishing Corporation.
8. Rose S, Bisson J. Brief early psychological interventions following trauma: a systematic review of the literature. Journal Traumatic Stress: 11, 697-710. 9. Campfield K. Mills A. Effect of timing of critical incident stress debriefing and post traumatic symptoms. Journal of Traumatic Stress 2001; 14.

10. Conlon L, Fahy T. Psychological debriefing a welcome demise. Ir J of
}

Psychol Med 2001; 18(2): 43-44

11. Bisson J, Jenkins L, Alexander J, Bannister C. Randomised controlled trial of psychological debriefing of victims of acute burns trauma. Br J Psychiatry 1999; 171 : 78-81.

\section{A case for transcranial magnetic stimulation}

Sir - We read with interest the editorial by O'Keane in September issue of the Irish Journal of Psychological Medicine ${ }^{1}$. We have recently treated eight patients meeting ICD-10 criteria for major depression with slow repetitive transcranial magnetic stimulation (rTMS) to the right prefrontal cortex. They volunteered for rTMS in preference to electroconvulsive therapy.

The small sample derived from both inpatients and outpatients provided written informed consent. The treatment protocol used was a replication of the protocol applied in the Klein Study 1999. ${ }^{2}$ Patients completed the Hospital Anxiety Depression Scale (HADS), the General Health Questionnaire-28 (GHQ-28) and the Symptom Checklist 90-R (SCL-90-R) on initial assessment. Progress was measured with the SCL-90-R.

Patients' medications remained constant and none was receiving ongoing psychotherapy at the time of treatment. Treatment was administered in ten daily sessions during a two-week period. A stimulation frequency of $1 \mathrm{~Hz}$ was used over two minutes as it is thought that low frequency rTMS may be less likely to induce seizures. ${ }^{3}$

Four out of the eight patients had greater than a $33 \%$ reduction in their depression T-Scores on the SCL-90-R and showed clinical signs of improvement. One of the patients with a dual diagnosis of major depression and Parkinson's disease had an improvement in both her depression scores and a 50\% reduction in her Parkinsonism, rated by the Webster Rating Scale.

Patient satisfaction level with the procedure was high. One patient withdrew midway through treatment due to feeling over stimulated and restless. With the exception of a headache in another patient, no other adverse side effects were described.

We agree with O'Keane that rTMS is "a valuable treatment option" and while it is not without risk it may be less stigmatising and pose fewer risks than electroconvulsive therapy. That being so it may find 'a clinical niche' in future.

\section{Ray Goggins \\ Specialist Registrar in General Adult and Old Age Psychiatry \\ Frenchay Hospital, Bristol BS16 1JB, England.}

References

1. O'Keane V. Trancrannial magnetic stimulation: an alternative physical treatment in depression. Ir J Psych Med 2001; 18(3): 79-81.

2. Klein E, Kreinin I, Chistyakov A et al. Therapeutic efficacy of right prefrontal slow repetitive transcranial magnetic stimulation in major depression. Arch Gen Psychiatry 1999; 56: 315-20.

3. Pascaul-Leone A, Valls-Sole J, Brasil-Neto JP et al. Seizure induction and transcranial magnetic stimulation. The Lancet 1992; 339: 997-9. 